\title{
Relocalización por dobles diferencias del cluster sísmico de Pisayambo
}

\author{
Double difference relocation \\ at Pisayambo seismic cluster
}

\author{
Sebastián Araujo*1, Liliana Troncoso² y Mario Ruiz² \\ I Centro de Investigación en Modelamiento Ambiental CIMA, Universidad Politécnica Salesiana, Quito, Ecuador. \\ 2 Instituto Geofisico de la Escuela Politécnica Nacional, Quito, Ecuador. \\ * Autor para correspondencia: jaraujo@ups.edu.ec
}

Nota: los datos utilizados en esta investigación fueron tomados del Instituto Geofísico de la Escuela Politécnica Nacional de Quito, Ecuador

\begin{abstract}
Resumen
En este artículo se presentan los resultados de la relocalización de sismos relacionados con el cluster sísmico de Pisayambo, provincia de Tungurahua en Ecuador. Se utiliza el programa hypoDD basado en el método de dobles diferencias. Los resultados obtenidos ofrecen evidencia sobre eventos en Pisayambo que definen una región caótica de focos sísmicos superficiales. Este trabajo es una muestra importante del funcionamiento del program hypoDD para el caso de problemas sísmicos altamente no lineales y subdeterminados.
\end{abstract}

Palabras clave: Pisayambo, sismología, dobles diferencias, hypoDD, problemas inversos.

\section{Summary}

This study reports the results of relocation of seismic events related to the seismic cluster of Pisayambo, in the Province of Tungurahua, Ecuador. The software hypoDD was used which is based in the method of double difference. The results thus obtained are evidence of the events in Pisayambo which define a chaotic region of seismic foci, all of them very superficial. This work shows the functioning of the hypoDD software in the case of highly nonlinear and underdetermined seismic problems.

Keywords: Pisayambo, seismology, double difference, hypoDD, inverse problems.

Forma sugerida de citar:

Araujo S., L. Troncoso y M. Ruiz. 2009. Relocalización por dobles diferencias del cluster sísimico de Pisayambo. La Granja. 10 (2). Pp. 27-34 ISSN: I390-3799. 


\section{Introducción}

La actividad sísmica del Ecuador presenta fenómenos interesantes conocidos como clusters, enjambres o nidos sísmicos. Éstos están constituidos por un gran número de eventos que se relacionan con un mismo fenómeno físico generativo. En el Ecuador se han detectado cuatro de estos clusters: al Norte de Quito, en Pujilí, en Pisayambo y en el Puyo. Cabe aclarar que la referencia a los lugares geográficos es puramente nominativa. De estos clusters, solamente el del norte de Quito ha merecido hasta la fecha un etudio profundo (Legrand et al., 2004 y 2002). En la actualidad se desarrollan estudios con mayor exhaustividad sobre el cluster de Pisayambo (Troncoso, 2009).

Específicamente, dentro del presente trabajo se analiza el cluster de Pisayambo que se ubica entre los $-0,9^{\circ}$ y $-1,3^{\circ}$ de latitud sur $y$ entre los $78^{\circ}$ y $78,5^{\circ}$ de longitud occidental, en virtud de que es el más activo y presenta la oportunidad para usarlo dentro de una tomografía del volcán Cotopaxi. Se ha bautizado con este nombre a un conjunto numeroso de sismos que se registran continuamente en la parte de la Cordillera de los Llanganatis que es frontera administrativa entre los cantones de Píllaro y Patate, donde se ubi- ca aproximadamente la laguna de Pisayambo. Para comprender la importancia de este fenómeno diremos que los sismos de Pisayambo aportan con el $40 \%$ de la actividad total registrada en la red de monitoreo del Instituto Geofísico de la Escuela Politécnica Nacional, IG (Aguilar et al., 1996).

Los datos utilizados para este trabajo provienen de los registros de las $6 \mathrm{I}$ estaciones de la red permanente del IG en los años 1996, 1997 y 1998. Además, se cuenta con los registros respectivos de una red temporal instalada en el volcán Cotopaxi con 17 estaciones (Monteiller et al., 2008). Esto nos permite tener un total de 8.093 eventos. Sobre estos eventos se ha determinado su onda $\mathrm{P}$ y onda $\mathrm{S}$ por piqueo manual de fases.

La primera localización de los eventos se ha hecho utilizando HYPOELLIPSE (Lahr, 1999). Para trabajar con este programa en el sistema operativo de disco DOS (Disk Operating System) se utilizó el modelo de velocidades estandarizado por el IG para el territorio del Ecuador (Troncoso, 2009), y que se muestra en la Tabla I; mientras que en la Figura I se puede ver la representación gráfica de todos los eventos lograda con la utilización del programa HYPOELLIPSE.

Tabla I.Velocidades de las ondas $\mathrm{P}$ en función de la profundidad de las cinco capas terrestres estudiadas. La cuarta columna hace referencia al coeficiente de la velocidad de la onda $\mathrm{P}$ sobre la velocidad de la onda $\mathrm{S}$, utilizado para la deducción de la velocidad de la onda S.

\begin{tabular}{cccc}
\hline Capa & $\begin{array}{c}V_{p} \\
(\mathrm{~m} / \mathrm{s})\end{array}$ & $\begin{array}{c}\text { Profundidad } \\
(\mathrm{km})\end{array}$ & $\mathrm{V}_{\mathrm{p}} / \mathrm{Vs}_{\mathrm{s}}$ \\
\hline $\mathrm{I}$ & 3,32 & 0 & 3,3 \\
2 & 5,9 & 5 & 3,3 \\
3 & 6,2 & 20 & 3,3 \\
4 & 6,7 & 30 & 3,3 \\
5 & 8,1 & 50 & 3,3 \\
\hline
\end{tabular}

\section{Materiales y métodos}

\section{I La relocalización por dobles diferencias}

La relocalización por dobles diferencias tuvo su origen en el estudio de los dobletes sísmicos: pares de microterremotos que tienen formas de onda similares, la misma localización hipocentral (punto al interior de la tierra, donde se inicia el movimiento sísmico) e idéntica magnitud, pero que son producidos en tiempos diferentes (Poupinet et al., 1984; Fréchet, 1985).
El método de relocalizar sismos por dobles diferencias se enmarca en el proceso previo necesario para, posteriormente, pasar a un estudio tomográfico. Efectivamente, antes de poder realizar una tomografía sísmica por dobles diferencias (Monteiller et al., 2005) se hace imprescindible relocalizar los sismos. Hay que señalar que si bien existe un trabajo previo de tomografía sísmica para el Ecuador (Monteiller et al., 2006) éste no hace uso todavía del método de dobles diferencias. El único intento anterior de relocalización por este método en sismos registrados en el territorio ecuatoriano se ha reali- 


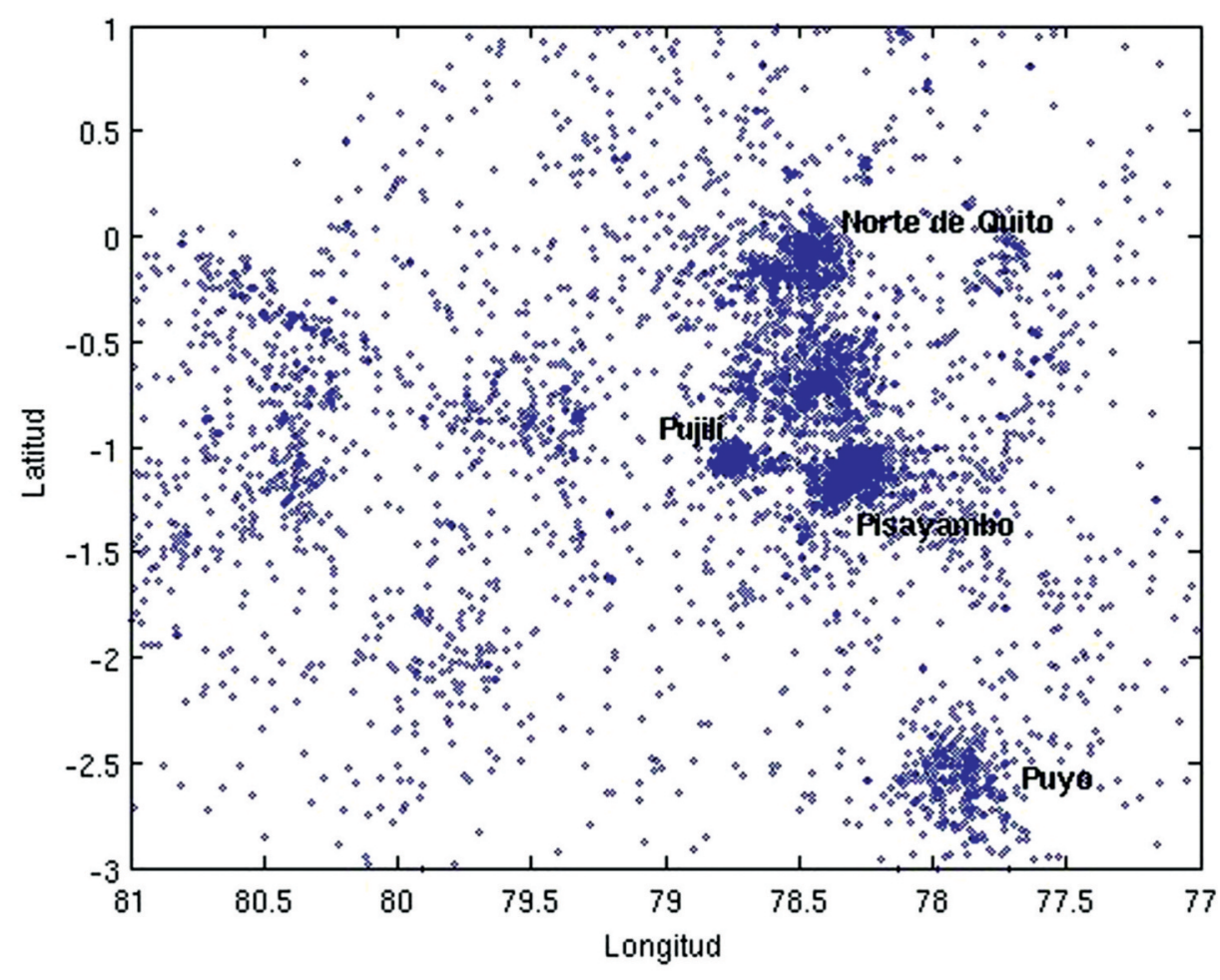

Figura I. Localización de la sismicidad de los años 1996, 1997 y 1998 obtenida con HYPOELLIPSE. Se puede observar la acumulación de sismos en los clusters del norte de Quito, Pujilí, Pisayambo y el Puyo. La mayor densidad de los sismos en el cluster de Pisayambo es evidente.

zado con eventos producidos en la falla de Esmeraldas (Elineau, 2008). Actualmente en el IG se vienen realizando pruebas con las dobles diferencias en el estudio de Pisayambo (Troncoso, 2009).

Para cumplir el objetivo planteado, se utilizó el programa hypoDD (Waldhauser, 200I). Esta serie de códigos en FORTRAN está sustentada por un algoritmo de relocalización que parte de los siguientes postulados (Waldhauser and Ellsworth, 2000): primero, poseemos una localización previa de los sismos que nos da una imagen de cluster; segundo, los sismos a ser relocalizados están generados por un mismo proceso físico. En una misma falla o conjunto cercano de fallas; tercero, la separación hipocentral entre dos eventos es pequeña comparada con la distancia entre el hipocentro del evento y la estación donde se registra; y por último, la separación hipocentral entre dos eventos es despreciable frente a las dimensiones de la estructura de las capas del modelo de velocidades utilizado en la localización previa.

Si estas cuatro condiciones se cumplen entonces la trayectoria de los rayos sísmicos entre sus hipocentros y la estación receptora son prácticamente idénticas y se puede conocer la separación relativa entre los eventos con gran precisión.

Partiendo de un par de sismos $i$ y $j$, registrados sobre la misma estación $k, t_{k}^{i}$ es el tiempo de arribo del i-ésimo sismo sobre la k-ésima estación, $t_{k}^{i}$ tiempo del j-ésimo sismo en la misma estación, la ecuación en dobles diferencias entre los tiempos observados y calculados se define como (Waldhauser and Ellsworth, 2000):

$$
d r_{k}^{i j}=\left(t_{k}^{i}-t_{k}^{j}\right)^{o b s}-\left(t_{k}^{i}-t_{k}^{j}\right)^{c a l}
$$

Ahora, si $\Delta m^{i}$ es la perturbación sobre el modelo de velocidades a través de la trayectoria $i, \Delta m^{i}$ es la perturbación de la trayectoria $j, y d r_{k}^{i j}$ la doble diferencia calculada entre los eventos $i$ y $j$, registrados en la misma estación $k$, podemos relacionar estos parámetros mediante la ecuación fundamental de la propagación de sismos en un medio:

$$
\frac{\partial t_{k}^{i}}{\partial m} \Delta m^{i}-\frac{\partial t_{k}^{j}}{\partial m} \Delta m^{j}=d r_{k}^{i j}
$$


Hasta ahora, se han tratado sólo dos eventos, pero se puede extender el mismo proceso a todas las parejas de eventos que queremos relocalizar. Para ello formamos un sistema de ecuaciones diferenciales que podemos escribir de forma matricial:

$$
W G m=W d(3)
$$

Donde $G$ es una matriz de dimensión $M^{*} 4 \mathrm{~N}$ con $\mathrm{M}$ el número de dobles diferencias observadas y $\mathrm{N}$ es el número de eventos que contiene las derivadas parciales, $d$ es el vector donde escribimos las dobles diferencias, $m$ es un vector de longitud $4 \mathrm{~N}$ que almacena los cambios en la posición hipocentral que queremos determinar, $y$ finalmente $W$ es una matriz diagonal que añade ponderaciones de cálculo a cada ecuación.

El objetivo es despejar el vector $m$ de la ecuación (3). El programa hypoDD realiza este trabajo utilizando un método de inversión linealizado conocido como los mínimos cuadrados ponderados. Es por esto la presencia de la matriz $W$ que contiene la calidad normalizada de los datos. Dicha calidad está cuantificada por un parámetro que puede variar entre cero y uno. Por tanto hypoDD resuelve iterativamente el sistema:

$$
m=\left(G^{T} W^{-1} G\right)^{-1} G^{T} W^{-1} d
$$

Este es un tipo de solución del problema inverso completamente lineal. Dado que el problema real, determinar la posición de los sismos en una estrucutura de velocidades no homogénea, es un problema inverso no lineal (Tarantola, 2005; Tarantola and Valette, 1982a; Tarantola and Valette, 1982b) el método $y$, por lo tanto, el programa hypoDD pueden tener limitaciones.

\subsection{Localización inicial del cluster de Pisa- yambo}

Previo a comenzar el proceso de relocalización definiremos las coordenadas donde se sitúan los eventos sísmicos de Pisayambo (Troncoso, 2009). Se define una región presentada en la Tabla 2 :

Tabla 2. Latitudes y longitudes donde se sitúan los eventos sísmicos de Pisayambo.

\begin{tabular}{|l|l|}
\hline Longitud máxima & $-0,9^{\circ}$ \\
\hline Longitud mínima & $-1,3^{\circ}$ \\
\hline Latitud máxima & $78^{\circ}$ \\
\hline Latitud mínima & $78,5^{\circ}$ \\
\hline
\end{tabular}

Esta región impone una separación máxima entre los sismos de $30 \mathrm{~km}$. Se escogen por tanto los sismos que caen dentro de estas coordenas lo que nos da un total de 1.525 eventos. Si vizualizamos las localizaciones proporcionadas por hypoellipse de estos sismos, lo que corresponde a un zoom sobre la Figura I, obtenemos el resultado mostrado en la Figura 2.

Mucho más grave es el resultado en profundidad que proporciona hypoellipse, muchos sismos se localizan sobre la superficie, y cuyos resultados se muestran en la Figura 3.

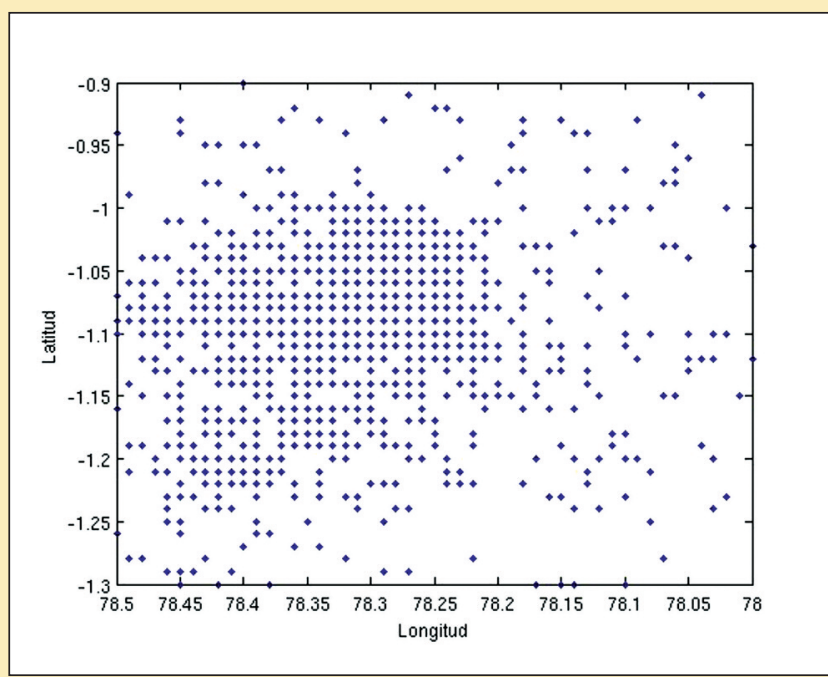

Figura 2. Localización inicial de los sismos del cluster de Pisayambo. Se constata la pobre definición lograda por este localización debido a que los sismos aparentan arreglos rectilíneos fruto de las limitaciones intrínsecas del programa hypoellipse.

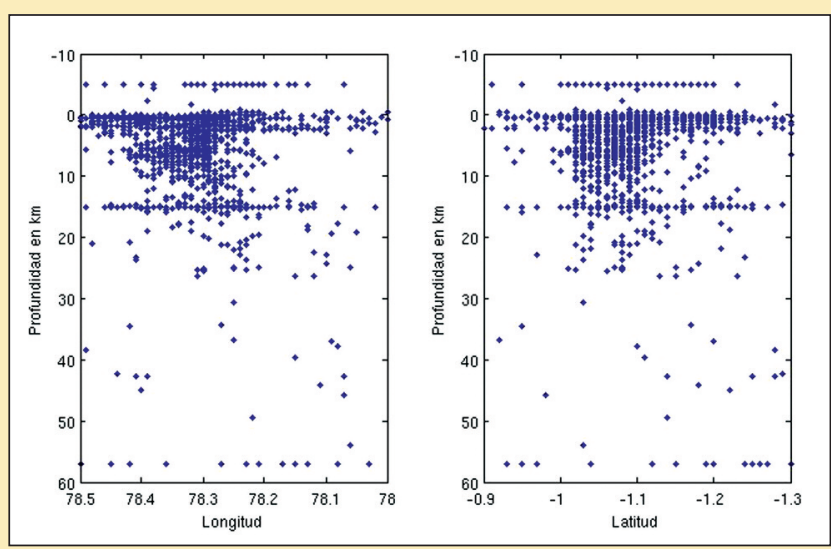

Figura 3. Localización en profundidad de los sismos del cluster Pisayambo proporcionados por hypoellipse. La superficie del terreno corresponde al 0 de profundidad. Los valores de profundidad negativa están sobre la superficie. La primera constatación válida es que los sismos de Pisayambo son eventos superficiales muy por encima del límite de las placas. 
De las Figuras 2 y 3 podemos concluir que si bien programas de localización previa como hypoellipse nos pueden dar una información inicial sobre la existencia de los clusters sísmicos, los mismos proporcionan información limitada sobre el fenómeno físico que los genera. En efecto, siendo que en la Figura 3 la mayoría de eventos sísmicos son superficiales y junto a otras consideraciones como el tipo mismo de sismicidad, llevaron a suponer que bien podría tratarse de un fenómeno volcánico antes que tectónico (Aguilar et al., 1996). Esta conclusión no ha podido ser corroborada en estudios más detallados, sobre todo, debido a la ausencia total de sismicidad de largo periodo propia de los ambientes volcánicos (Troncoso, 2009).

Si aceptamos que la sismicidad de Pisayambo está relacionada con eventos de fallas superficiales como lo sugieren estudios geológicos y geográficos (Eguez et al., 2003), se hace necesario obtener la imagen sísmica de dichas fallas. De ahí la importancia de la utilización del programa hypoDD que está enfocado al tratamiento de la sismicidad de este tipo de fenómenos.

\subsection{Aplicación de hypoDD a los eventos del cluster de Pisayambo}

El primer paso consiste en disponer la información sobre los eventos en el formato de lectura adecuado para hypoDD. Los archivos de entrada necesarios para hypoDD son: longitud y latitud de las estaciones sísmicas, posición inicial de los eventos y sus diferencias de tiempo absolutas para cada esta- ción. Las diferencias de tiempo absolutas se calculan como:

$$
\text { dif }=\left|t_{l o c}-t_{o b s}\right|
$$

Donde el valor absoluto de la diferencia se toma entre el tiempo proporcionado por la localización inicial y el tiempo observado en cada evento.

Utilizamos el programa ph2dt (Waldhauser, 200I) para formar las diferencias entre los tiempos de arrivo para cada par de eventos del cluster. Este programa necesita definir los siguientes parámetros iniciales:

MINWGHT : ponderación mínima permitida para la calidad de fases en los eventos.

MAXDIST: máxima separación en kilómetros entre un par de eventos y la estación donde se registran.

MAXSEP : separación máxima en kilómetros entre los hipocentros de los eventos.

MAXNGH: número máximo de eventos vecino entre los que se calcularán las diferencias de tiempos de arrivo.

MINLNK: número mínimo de fases ligadas necesarias para definir la vecindad de un evento.

MINOBS: número mínimo de fases ligadas que se conservarán para cada par de eventos.

MAXOBS: número máximo de fases ligadas que se conservarán para cada par de eventos.

En nuestro caso usamos los siguientes parámetros para ph2dt (Tabla 3):

Tabla 3. Parámetros utilizados en el programa ph2dt.

$\begin{array}{ccccccc}\text { MINWGHT } & \text { MAXDIST } & \text { MAXSEP } & \text { MAXNGH } & \text { MINLNK } & \text { MINOBS } & \text { MAXOBS } \\ 0 & 500 & 6,2 & 10 & 8 & 8 & 78\end{array}$

Con estos parámetros, el programa ph2dt nos permite rescatar 1.105 eventos formando 2.614 pares de eventos con un total de 23.5 I I pares de fases. Así, el fichero generado por ph2dt se utiliza como entrada para el programa hypoDD. Lo primero que hay que advertir es que hypoDD puede procesar variados tipos de fases: ondas $\mathrm{P}$, ondas $\mathrm{S}$, correlación espectral cruzada de ondas $\mathrm{P}$, correlación espectral cruzada de ondas $S$ y todas las anteriores en conjunto. Para nuestro estudio utilizaremos únicamente la información del piqueo de fases de las ondas $P$.

Antes de proceder a correr hypoDD se debe advertir que el momento de compilar este progra- ma se debe escoger el tamaño del cluster que se quiere estudiar. Existen tres opciones: problemas pequeños (decenas de eventos), problemas medianos (centenas de eventos) y problemas grandes (miles de eventos). En nuestro caso escogemos la opción de problemas grandes la cual se realiza en el archivo hypoDD.inc y luego se procede a recopilar el programa. La elección del tamaño del problema también impone condiciones en la capacidad de cálculo de la máquina disponible. Se corrió hypoDD en una laptopt ACER ASPIRE $4730 Z$ con un procesador Intel doble núcleo con $2.16 \mathrm{GHz}$ velocidad y una memoria RAM de 2.8 GB. Bajo estas condicio- 
nes hypoDD procesa los 1.105 eventos en menos de tres segundos.

Para su funcionamiento hypoDD impone la elección de una serie de parámetros de los cuales detatallaremos los que son útiles en nuestro estudio:

OBSCT: número mínimo de observaciones para cada par usado para saber si forma parte del cluster. Usamos 10 que coinciden con el número máximo de vecinos MAXNGH.

NSET: número del conjunto de iteraciones que van a ser usadas en la inversión. Planteamos dos conjuntos de iteraciones. El segundo conjunto permite obtener mayor precisión en los hipocentros proporcionados después de correr el primer conjunto.

NITER: número de iteraciones para los cuales corre la inversión. En cada conjunto se proponen 8 iteraciones. En total, por lo tanto, hay 16 iteraciones.

WTCTP: peso asignado a la calidad de las ondas P. Dado que nuestros datos son de peso uniforme asignamos a todos los eventos el peso máximo $I$.

WDCT: máxima distancia en km entre los dos sismos que forman un par. Es el parámetro más delicado para asegurarse de que hypoDD logre obtener resultados relocalizando los eventos. Después de varias pruebas numéricas WDCT es tomado en su máximo valor posible para nuestro problema: $6,2 \mathrm{~km}$. Con distancias mayores se produce un escalamiento en la matriz de inversión e hypoDD no logra resolver el problema.

Hay que agregar también como parámetros la estrucutura del modelo de velocidades que ya fue explicitada anteriormente. El resto de parámetros de hypoDD se toman en los valores sugeridos para problemas con un gran número de sismos (Waldhauser, 200I).

El resultado de la relocalización de hypoDD se presenta en la Figura 4. Aquí vemos cuatro paneles. En los dos paneles superiores se muestran: la posición de las estaciones y por lo tanto las coordenadas geográficas del cluster total, y la misma figura pero con los sismos relocalizados y en lugar de coordenadas geográficas las distancias en $\mathrm{km}$. La salida gráfica de hypoDD también nos permite realizar cortes a través de las direcciones AA' y BB' para observar la localización de los eventos en profundidad. Esto es lo que se muestra en los paneles inferiores. El máximo número de eventos que logra relocalizar hypoDD es 137.
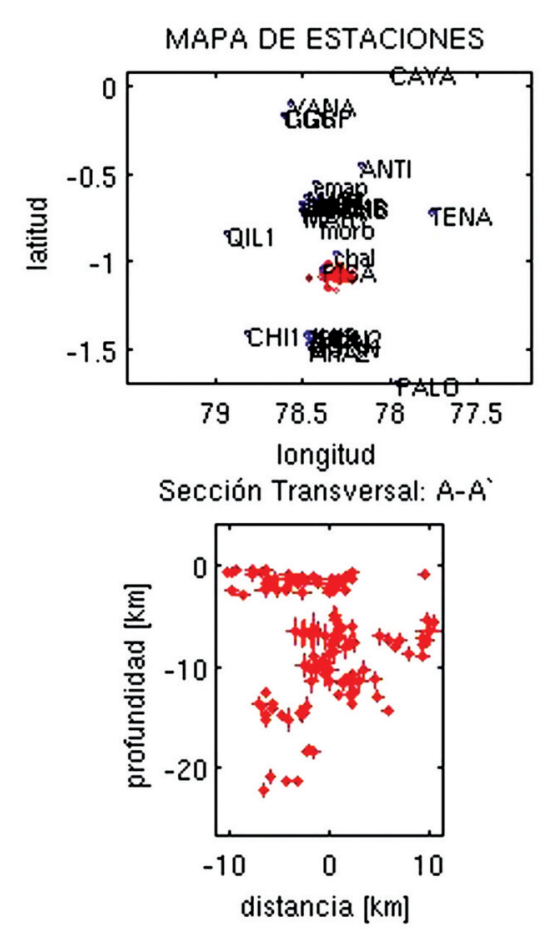

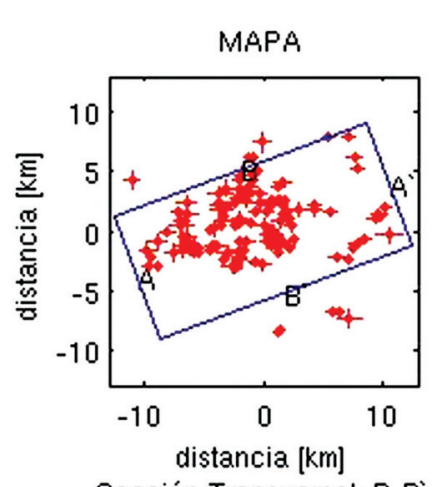

Sección Transuersal: B-B'

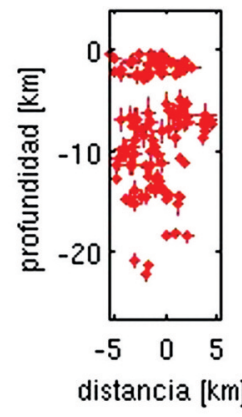

Figura 4. Salida gráfica proporcionada por hypoDD. Los eventos corresponden a puntos de color rojo con barras de error en las tres dimensiones. En el primer panel se muestran todas las estaciones que el programa ha utilizado. Se resalta una gran acumulación de estaciones al norte del cluster que se ubican en el volcán Cotopaxi y al sur del cluster donde se halla el volcán Tungurahua. En el segundo panel se muestran todos los sismos relocalizados. El tercero y cuarto panel muestran secciones en profundidad en las direcciones A-A ${ }^{\prime}$ y B-B'. 


\section{Discusión}

Aunque pueda parecer limitado, en principio, que hayamos logrado la relocalización de 137 sismos de un grupo inicial de I.525, hay que tomar en cuenta que el principal objetivo del uso de hypoDD es poder definir los clusters partiendo de criterios incontrovertibles para su existencia. Con esto queremos decir que, si los eventos sísmicos que estamos analizando no cumplen con los postulados que se señalaron anteriormente, hypoDD no hubiera proporcionado ningún resultado.

Hay que repetir que el principal punto de partida para la localización en dobles diferencias es tener eventos ligados entre sí. Si esto no se cumple, la matriz $\boldsymbol{G}$ de la ecuación (3) resultará mal condicionada y la solución se tornará inestable. Una forma de asegurarse eventos ligados es la utilización previa del programa ph2dt. Este programa, recordamos, requiere definir un mínimo número de observaciones (MINOBS) que a su vez depende de la distribución geométrica de las estaciones que registren el par de eventos (Waldahauser and Ellsworth, 2000).Y es aquí donde surge el principal inconveniente que en nuestro caso no nos permite explotar todas las capacidades de hypoDD. Si nos remitimos al primer panel de la Figura 4, donde podemos observar la distribución geométrica de las estaciones que registran los eventos del cluster Pisayambo, observamos que sólo poseemos dos estaciones (PISA y chal) situadas exactamente sobre el cluster.Además estas dos estaciones no aseguran una correcta cobertura azimutal por estar situadas en la misma zona geográfica. El resto de nuestras estaciones donde se registran los eventos del cluster, 27 estaciones adicionales, se encuentran a una considerable distancia del fenómeno lo que implica que las formas de onda de los simos de Pisayambo son afectadas por la gran distancia que recorren antes de ser registradas.

Este inconveniente se puede solucionar de dos maneras. La primera, experimental, consiste en instalar estaciones alrededor del cluster Pisayambo para asegurarse una correcta cobertura azimutal. Este paso ya ha sido dado por el IG al llevar a cabo un experimento que consistió en instalar cuatro estaciones sísmicas alrededor de Pisayambo. Los resultados de este experimento se encuentran todavía en fase de estudio (Troncoso, 2009).

La segunda forma en que podemos incrementar los resultados de relocalización por dobles diferencias usando únicamente los datos del presente artículo, consiste en reconocer que el resolver la ecuación (3) implica un problema inverso no lineal y subdeterminado.
La linealidad del programa hypoDD se manifiesta cuando al resolver iterativamente el sistema (4), se hace la suposición de que si añadimos una perturbación_ $m$ al vector $m$ que contiene los parámetros que deseamos descubrir, en nuestro caso las localizaciones hipocentrales, se debe cumplir junto a la matriz de derivadas parciales $G$ que representan nuestra relación funcional:

$$
G(m+\Delta m)=G(m)+G(\Delta m)
$$

Para comprender lo forzado de la ecuación (5) recordemos que $G$, incluye las derivadas parciales de los tiempos de propagación de los eventos respecto a sus coordenadas. Si $G$ fuese lineal, esto querría decir que si conocemos con precisión la localización de un evento y quisiéramos averiguar el tiempo de propagación del mismo evento pero desde una posición hipocentral ligeramente perturbada respecto a la posición original, tan sólo tendríamos que calcular el tiempo de propagación entre la nueva posición y la inicial y sumarla al tiempo primero. Todo esto sería cierto si consideramos que el medio por donde se propaga el sismo tiene una velocidad homogénea. Pero el medio geofísico natural es altamente heterogéneo y nada nos garantiza que si nos movemos un poco de la posición inicial nos vamos a encontrar con el mismo tipo de medio. Lógicamente siempre esperamos que la naturaleza sea gentil y que cuando perturbemos infinitesimalmente el hipocentro de partida, el medio continúe siendo el mismo.

El otro problema, la subdeterminación, en nuestro caso implica que tenemos más incógnitas que ecuaciones a resolver, es decir tenemos muy pocas estaciones sísmicas y muy mal distribuidas. Tómense en cuenta que las pruebas iniciales de hypoDD fueron hechas en la región de California donde existen no cientos sino miles de estaciones símicas en total cobertura azimutal del fenómeno que se estudia.

Para resolver problemas inversos altamente no lineales y subdetermidandos contamos actualmente con aproximaciones teóricas válidas (Tarantola and Valette, 1 982a; Tarantola and Valette , 1982b) cuya aplicación y comparación con los resultados de este artículo será el interés de trabajos posteriores.

\section{Conclusiones}

A pesar de todas las limitaciones anteriores, el uso del programa de relocalización hypoDD en los eventos del cluster Pisayambo permite una primera visión de su estructura. En el segundo panel de la Figura 4, 
se observa que el cluster se extiende en una zona de $22,5 \mathrm{~km}$ de largo por $5,5 \mathrm{~km}$ de ancho en una dirección que forma un ángulo de $\mathrm{N} 70^{\circ} \mathrm{E}$.

En el tercer panel se aprecia el corte en la dirección principal del cluster A-A'. Se definen pequeños sub-clusters. El primero, muy superficial, a menos de $2 \mathrm{~km}$ de profundidad. Luego algunos más entre 3 y $15 \mathrm{~km}$ de profundidad. Esto confirma una vez más que los eventos de Pisayambo no están ligados a la actividad de colisión tectónica que se encuentra mucho más profunda, por lo menos a $30 \mathrm{~km}$. La relación de estos clusters con fallas activas y una mejor definición de los mismos, todavía son un reto para investigaciones futuras tanto en la geología como en la geofísica.

\section{Agradecimientos}

El presente trabajo se realizó dentro del marco de los proyectos de investigación financiados por la Universidad Politécnica Salesiana, con el apoyo especial del Área de Ciencias de la Vida. Un reconocimiento especial a Diego Villegas y Javier Vera estudiantes de la Universidad Politécnica Salesiana por su ayuda en llevar al Linux OpenSuse a su óptimo nivel de funcionamiento. Los datos RENSIG son los que se utilizan en este estudio.

\section{Referencias}

Aguilar J., Chatelain Jean-Luc, Guillier Bertrand, Yepes H.1996. The Pisayambo, Ecuador, seis-micity nest: towards the birth of a volcano? Géodynamique andine, résumés étendus. Andean geodynamics, extended abstracts. Paris. ORSTOM. 1996, Pp. I25- I 28. (Colloques et Séminaires). ISAG 96 : Symposium International sur la Géodynamique Andine, 3., Saint-Malo (FRA), 1996/09/17-19.

Eguez A.,A.Alvarado, H.Yepes, M. N.Machette, C. Costa y R.L. Dart. 2003. Database and Map of Quaternary faults and folds of Ecuador and its offshore regions, USGS, Open-File Report 03-289.

Elineau, S. 2008. Relocalisation d'un essaim de séismes et mécanisme de déformation sur la marge active d'Equateur, Master ler année «Sciences et Gestion de la Terre». Direction: Emmanuel TRIC, Université NiceSophia Antipolis.
Fréchet, J. 1985. Sismogenèse et doublets sismiques. Thèse d'Etat, Université Scientifique et Médicale de Grenoble, 206 pp.

Lahr, J. 1999. HYPOELLIPSE: A Computer Program for Determining Local Earthquake Hypocentral Parameters, Magnitude, and First-Motion Pattern (Y2K Compliant Version).Version I.0, U. S. Geological Survey Open-File Report 99-23, On-Line Edition.

Monteiller,V., J.P. Métaxian, B. Valette,S. Araujo. 2008. Seismic tomography of the Cotopaxi volcano, Ecuador. 7th International Symposium on Andean Geodynamics (ISAG 2008, Nice). Extended Abstracts: 339-340.

Monteiller, V., S. Araujo, J.P. Métaxian, B. Valette. 2006. Tomografía de ondas $\mathbf{P}$ en el Ecuador. $\mathrm{VI}$ Jornadas en Ciencias de la Tierra. Escuela Politécnica Nacional. Quito, Ecuador.

Monteiller,V., JL. Got, J.Virieux, P. Okubo. 2005. An efcient algorithm for Doubble diference tomography and location in heterogeneous media, with application to the $\mathrm{Ki}$ lauea volcano. J. Geophys. Res., I I O, BI 2306.

Poupinet, G.,W. L. Ellsworth, and J. Fréchet. I984. Monitoring velocity variations in the crust using earthquake doublets: an application to the Calaveras fault, California, J. Geophys. Res. 89, 5719-573I.

Tarantola, A. 2005. Inverse Problem Theory and Methods for Model Parameter Estimation, SIAM.

Tarantola A., B.Valette. 1982a. Generalized non linear inverse problems solved using the leastsquares criterion. Rev. Geophysics. 20, 219232

Tarantola A., B.Valette. 1982b. Inverse Problems=Quest for Information. J.Geophysics. 50, 159170.

Troncoso, L. 2009. Sismicidad del enjambre sísmico de Pisayambo. Tesis de Maestría. Université de Nice-Sophia Antipolis-Escuela Politécnica Nacional (en curso de realización).

Waldhauser, F. 200I. hypoDD - A Program to Compute Double-Difference Hypocenter Locations (hypoDD version I.0 03/200I). U.S. Geol. Survey Open File Report $0 \mathrm{I}-\mathrm{II} 3$.

Waldhauser, F., and W. L. Ellsworth. 2000. A double diference earthquake location algorithm: Method and application to the northern Hayward fault, CA, Bull 